\title{
Revue bibliographique : caractérisation de la thermorésistance des spores bactériennes pour l'optimisation des traitements UHT
}

\author{
O. CERF
}

I.N.R.A., Laboratoire de Génie des Procédés alimentaires 25, avenue de la République, 91300 Massy, France

\begin{abstract}
Résumé
On compare les avantages et les difficultés des techniques de mesure de thermorésistance des spores dans les conditions du traitement UHT ou dans des conditions destinées à les simuler. Ces techniques font appel à des souches de laboratoire ou à des flores sporulées naturelles, chauffées soit dans des tubes, soit dans des appareils UHT maquettes ou de dimensions industrielles. Pour l'optimisation des traitements UHT en usine, on recommande de caractériser les flores naturelles chauffées dans les installations industrielles de chaque usine.
\end{abstract}

Mots clefs : Spores bactériennes - Stérilisation UHT - UHT - Lait.

\section{Summary}

Inactivation kinetics of bacterial spores and UHT process optimization

Measurement techniques of heat resistance of spores were compared. Advantages and difficulties of using natural spore flora versus laboratory strains and of using industrial heating versus pilot plant, were discussed. For optimizing industrial UHT treatments, characterization of milk natural spore flora heated in the actual industrial plant of each factory is recommended.

Key words: Bacterial spores - UHT sterilization - UHT - Milk.

Note : ce texte reprend une conférence faite lors d'une réunion du Cercle international des chercheurs laitiers à Kiel en 1984, à la demande du Professeur H. Reuter. 


\section{Introduction}

Associé au conditionnement aseptique, le traitement thermique en écoulement continu à ultra-haute température (UHT) - c'est-à-dire à plus de $135^{\circ} \mathrm{C}$ pendant quelques secondes - a pris une importance industrielle considérable pour la fabrication de lait de longue conservation.

La réglementation prévoit que le lait UHT doit rester stable. Il doit donc avoir été débarrassé par le traitement thermique des spores bactériennes susceptibles de le déstabiliser. Un grand nombre de travaux de recherche ont porté sur la destruction des spores dans des conditions de laboratoire. Ils utilisaient des spores de souches pures sélectionnées pour leur thermorésistance élevée, produites dans des milieux de culture autres que le lait, et dénombrées en général dans des milieux de culture, plus rarement dans le lait. En général, les conditions de traitement thermique étaient différentes des conditions de traitement réelles. Toutefois, certains travaux ont été réalisés dans des appareils UHT maquettes ou pilotes, en écoulement continu ou non. Enfin, des essais récents ont porté sur l'inactivation des spores du lait lui-même, chauffées en conditions industrielles et dénombrées dans le lait.

Le présent article se propose de présenter une revue critique des résultats publiés jusqu'ici, et d'en tirer une conclusion pratique, de façon à aider les industriels laitiers dans leurs décisions.

On examinera successivement le choix des microorganismes, les méthodes de mesure de la thermorésistance et l'interprétation des résultats.

\section{Choix des microorganismes}

Est-il préférable d'étudier 1a flore sporulée naturelle du lait, ou des souches isolées, cultivées en laboratoire ? Est-il préférable d'étudier les spores de bactéries mésophiles, ou celles de bactéries thermophiles?

\section{A. Flore naturelle}

Une méthode approximative d'une grande simplicité a été proposée par Ashton (1966) pour étudier la flore sporulée dans le lait d'une usine donnée et choisir la température de stérilisation.

La voici :

- stériliser le lait à des températures décroissant par pas de 3 à $6 \mathrm{~K}$ (K est le symbole du kelvin, unité SI à utiliser pour les écarts de températures et pour les températures absolues);

- prélever un échantillon de 250 unités conditionnées aseptiquement;

- en incuber la moitié 15 jours à $22^{\circ} \mathrm{C}$, l'autre 10 jours à $37^{\circ} \mathrm{C}$;

- prendre comme température de stérilisation optimale $3 \mathrm{~K}$ au-dessus de la température la plus basse à laquelle l'échantillon ne comporte aucun défec- 
tueux. En aucun cas ne choisir une température inférieure à $138^{\circ} \mathrm{C}$;

- répéter l'expérimentation tous les 3 mois.

Une méthode plus sophistiquée a été proposée par HoraK (1980) :

- les spores du lait matière première sont dénombrées par une technique conventionnelle ;

- après traitement UHT, des échantillons de lait sont incubés à 30 ou $55^{\circ} \mathrm{C}$, et, s'il y a déstabilisation du lait, la présence de bactéries sporulantes est vérifiée par les techniques microbiologiques habituelles;

- au moyen d'une technique statistique, les temps de réduction décimale D sont calculés à plusieurs températures, ainsi que la valeur de z.

Cette méthode a été modifiée par ODE t et al. (1985) qui, pour les spores du lait matière première, dénombrent seulement celles qui sont susceptibles de se développer dans le lait. L'article de DuQuet et al. (1986) présente des résultats obtenus ainsi, résumés dans le tableau I, et fournit les détails pratiques de la méthode.

\section{B. Souches de laboratoires}

Les souches de laboratoires ne peuvent servir que si leur thermorésistance est complètement connue, c'est-à-dire si leurs courbes de survies ont été établies à plusieurs températures.

Le tableau I donne la liste des souches étudiées par différents auteurs. Toutefois, quelques références ne sont pas citées dans le tableau. Il s'agit de MARTIN et Harper (1964) (travaillant avec 9 souches) et de Torres et Hedrick (1969), qui ont seulement vérifié si toutes les spores chauffées étaient inactivées ou pas, en fonction de la température.

Le tableau I montre qu'en général les souches de laboratoire ne sont pas représentatives des flores naturelles. Celles pour lesquelles les valeurs de z (paramètre défini au paragraphe III-B-2) sont les plus voisines des valeurs des flores naturelles sont les souches utilisées par Hermier et al. (1959), Burton et al. $(1958,1959,1977)$ et Konietzko et Reuter (1980). Mais leurs temps de réduction décimale sont 5 à 10 fois plus grands. Leurs courbes d'inactivation présentent en outre un ou plusieurs des défauts suivants :

- épaulement, correspondant à la nécessité d'une activation par la chaleur ;

- traînée ou concavité vers le haut.

\section{Discussion}

Il ressort de la comparaison précédente que les souches de laboratoire utilisées jusqu'ici ne sont pas représentatives des populations naturelles de spores. Pour celles où la température influe de la même façon sur leur destruction ( $\mathrm{z}$ voisins), les souches de laboratoire ont une thermorésistance si élevée que leur utilisation pour élaborer des barèmes de stérilisation conduirait à un surchauffage excessif du lait. L'étude des flores naturelles semble donc plus avantageuse pour les industriels. Faite avec les techniques indiquées plus haut, elle porte précisément sur les spores susceptibles de se développer dans le lait lui-même après traitement UHT, les seules dont l'industriel a à se préoccuper (voir aussi paragraphe II-C). 
TABLEAU I

\begin{tabular}{|c|c|c|c|}
\hline Références & Micro-organismes & $\underset{(\mathrm{s})}{\mathrm{D} 121^{\circ} \mathrm{C}}$ & $\stackrel{\mathrm{z}}{(\mathrm{K})}$ \\
\hline $\begin{array}{l}\text { HORAK }(1980) \\
\text { HORAK (1980) } \\
\text { DUQUET et al. (1986) }\end{array}$ & $\begin{array}{l}\text { Flore naturelle mésophile } \\
\text { Flore naturelle thermophile } \\
\text { Flore naturelle thermophile }\end{array}$ & $\begin{array}{l}11 \\
25 \\
58\end{array}$ & $\begin{array}{r}10,4 \\
10,4 \\
9,6\end{array}$ \\
\hline $\begin{array}{l}\text { GALESLOOT (1956) } \\
\text { BURTON et al. (1957-59) } \\
\text { BURTON et al. }(1957-59) \\
\text { BOTTAZZI et al. }(1966) \\
\text { BOTTAZZI et al. }(1966) \\
\text { HERMIER et al. (1959) } \\
\text { LINDGREN et al. }(1963) \\
\text { SRIMANI et al. }(1980)\end{array}$ & $\begin{array}{l}\text { Bacillus subtilis sp. } \\
\text { Bacillus subtilis } 786 \text { subpop. } 1 \\
\text { Bacillus subtilis } 786 \text { subpop. } 2 \\
\text { Bacillus subtilis } 786 \\
\text { Bacillus subtilis CaIMP } \\
\text { Bacillus subtilis SJ2 } \\
\text { Bacillus subtilis } 736 \\
\text { Bacillus subtilis sp. }\end{array}$ & $\begin{array}{l}\overline{21} \\
\overline{90} \\
\overline{1} \overline{120} \\
\overline{0,001}\end{array}$ & $\begin{array}{r}\overline{6,7} \\
6,7 \\
\overline{-} \\
\overline{10,5} \\
\overline{3,6}\end{array}$ \\
\hline $\begin{array}{l}\text { BURTON et al. (1957-59) } \\
\text { BURTON et al. (1977) } \\
\text { HOSETTLER et al. (1961) } \\
\text { LINDGREN et al. (1963) } \\
\text { BUSTA (1967) } \\
\text { CERF et al. (1973) } \\
\text { NEAVES et al. (1978) } \\
\text { SHIH et al. (1982) } \\
\text { WANG et al. (1964) } \\
\text { OQUENDO et al. (1975) } \\
\text { SRIMANI et al. (1980) } \\
\text { KONIETZKO et al. (1980) }\end{array}$ & $\begin{array}{l}\text { B. stearothermophilus TH24 } \\
\text { B. stearothermophilus TH24 } \\
\text { B. stearothermophilus } 1518 \\
\text { B. stearothermophilus } 1518 \\
\text { B. stearothermophilus } 1518 \\
\text { B. stearothermophilus } 1518 \\
\text { B. stearothermophilus } 1518 \\
\text { B. stearothermophilus } 1518 \\
\text { B. Stearothermophilus } \mathrm{FS} 7954 \\
\text { B. stearothermophilus } \mathrm{sp} \\
\text { B. stearothermophilus } 9372 \\
\text { B. stearothermophilus } \mathrm{C} 953\end{array}$ & $\begin{array}{l}233 \\
360 \\
- \\
140 \\
440 \\
420 \\
89 \\
10 \\
10 \\
10 \\
95\end{array}$ & $\begin{array}{c}9,43 \\
8,73 \\
- \\
\overline{6,7} \\
7,5 \\
7 \text { à } 7,4 \\
8,3 \\
8 \\
5,6 \\
6,4 \\
10,8\end{array}$ \\
\hline HERMIER et al. (1975) & B. coagulans 604 & 60 & 7 \\
\hline $\begin{array}{l}\text { Pflug et al. (1953) } \\
\text { Busta (1967) }\end{array}$ & $\begin{array}{l}\text { Clostridium PA } 3679 \\
\text { Clostridium PA } 3679\end{array}$ & $\begin{array}{r}64 \\
7\end{array}$ & $\begin{array}{r}9,3 \\
19,4\end{array}$ \\
\hline $\begin{array}{l}\text { NEAVES et al. (1978) } \\
\text { NEAVES et al. (1978) }\end{array}$ & $\begin{array}{l}\text { C. botulinum type A } \\
\text { C. botulinum type B }\end{array}$ & $\begin{array}{l}3 \\
3\end{array}$ & $\begin{array}{l}6,5 \text { à } 56 \\
6,9 \text { à } 61\end{array}$ \\
\hline
\end{tabular}

Pour savoir s'il est préférable de tenir compte des bactéries sporulées thermophiles plutôt que des bactéries sporulées mésophiles, il faut pouvoir répondre successivement aux questions suivantes :

1. Le produit fini risque-t-il de séjourner à des températures supérieures à $45^{\circ} \mathrm{C}$ (pays tropicaux, ou hangars ou camions exposés au soleil dans les pays tempérés)?

2. Si oui, le lait contient-il des spores de thermophiles ?

3. Si oui, leur concentration est-elle élevée au point que, en raison de leur thermorésistance, il soit nécessaire de concevoir le traitement thermique pour elles et non pour les mésophiles?

Si les réponses sont toutes positives, l'importance des spores de thermophiles devient primordiale. 


\section{Méthodes expérimentales pour les souches en laboratoire}

Les flores naturelles présentent l'inconvénient d'être des populations mélangées. Elles se prêtent mal à l'étude détaillée des procédés par des méthodes scientifiques rigoureuses. C'est pourquoi les souches pures gardent une utilité certaine dans de nombreux cas. Cette section passe en revue les difficultés des méthodes expérimentales utilisées pour les souches de laboratoire.

\section{A. Préparation des spores}

La sporulation n'est pas toujours facile à obtenir. Les lots de spores peuvent être différents les uns des autres. Certains ont besoin d'être activés par la chaleur pour atteindre leur thermorésistance maximale. D'autres se modifient durant le chauffage et deviennent davantage thermorésistants.

\section{Activation}

Beaucoup de souches doivent être activées, en particulier les plus thermorésistantes :

- Bacillus subtilis SJ2 (Hermier et al., 1959);

- B. stearothermophilus TH24 (BuRTON et al., 1959);

- B. stearothermophilus 1518 (Cerf et Hermier, 1973 ; Shin et al., 1982).

Avec B. stearotermophilus 9372, SRImani et al. (1980) ont fait une observation curieuse : les spores activées par la chaleur résistent davantage à la chaleur.

\section{Préchauffage}

SPECK et Busta (1968) ont observé que les spores de la souche B. stearothermophilus 1518 préchauffées $60 \mathrm{~min}$ à $90^{\circ} \mathrm{C}$ (donc non activées) deviennent plus thermorésistantes à $121^{\circ} \mathrm{C}$.

\section{Reproductibilité}

Beaucoup de travaux de recherches peuvent être faits avec un seul et même lot de spores. Mais, s'il faut utiliser un second lot, le manque de reproductibilité pose un problème. A titre d'exemple, on peut citer BuRToN et al. (1977) qui trouvaient pour TH24 des valeurs de D à $120^{\circ} \mathrm{C}$ allant de 660 à $990 \mathrm{~s}$, ou CERF (1979) qui, tout en trouvant des valeurs proches de $\mathrm{D}$ à $120^{\circ} \mathrm{C}$, comprises entre 2,1 et $2,4 \mathrm{~s}$, trouvait que $\mathrm{z}$ variait de 6,1 à $8 \mathrm{~K}$ pour $B$. coagulans 604 .

Du fait que B. stearothermophilus 1518 est très utilisé comme bio-indicateur pour le contrôle des stérilisateurs notamment dans le domaine hospitalier, le problème de la reproductibilité a fait l'objet de nombreuses recherches. Il en ressort que la forme de l'inoculum (spores ou cellules végétatives), la composition du milieu de sporulation, les températures d'incubation de l'inoculum et du milieu de sporulation, l'oxygénation lors de la sporulation, sont parmi les facteurs importants de la reproductibilité. 


\section{B. Technique de chauffage}

\section{Tubes} l'esprit.

Le chauffage en tubes est la technique qui se présente la première à

En dehors des tubes à essai ordinaires, on utilise le plus couramment deux types de tubes :

- les tubes de TDT (temps de destruction thermique) en verre ou en métal (cuivre ou inox) ont un diamètre intérieur de 0,5 à $1 \mathrm{~cm}$, et contiennent de 1 à $2 \mathrm{~cm}^{3}$ de suspension de spores. Leurs temps d'équilibrage à la température du bain sont de 1 à $2 \mathrm{mn}$. Ils sont faciles à utiliser;

- les tubes capillaires en verre ont un diamètre intérieur de 0,5 à $1 \mathrm{~mm}$, et contiennent de 10 à $100 \mathrm{~mm}^{3}$ de suspension. Leur temps d'équilibrage est en général inférieur à $10 \mathrm{~s}$. Ils sont moins faciles à utiliser que les tubes de TDT, mais présentent l'avantage de pouvoir être employés à des températures plus élevées (grâce à leur équilibrage rapide, on peut les utiliser à des températures où l'inactivation des spores est, elle aussi, rapide). Burton et al. (1977) ont fait des mesures de haute précision dans des capillaires et indiquent des techniques de calcul permettant d'exploiter les résultats pour des chauffages très courts, de limite inférieure $2 \mathrm{~s}$.

\section{Appareils UHT}

Le chauffage dans un appareil UHT est la technique qui semble la plus réaliste. Il existe deux manières de la mettre en cuvre :

a. Avec des appareils spécialement conçus. Le plus ancien est le Thermoresistomer, utilisé par exemple par Pflug et Esselen (1953).

On peut citer également :

- MISTRESS, initiales d'une définition anglaise dont la traduction est Equipement de recherche à échelle réduite pour le traitement du lait par injection dans la vapeur (PERKIN, 1974);

- l'appareil utilisé par DAUDin et CERF (1977).

Les trois appareils ci-dessus mettent en contact les spores avec de la vapeur vive. L'appareil de WANG et al. (1964) comme ceux utilisés par OQUENDo et al. (1975) et SRIMANi et al. (1980) assurent le mélange de la suspension de spores avec un excès de liquide chaud.

b. Avec des appareils UHT en écoulement continu, directs ou indirects, expérimentaux ou industriels, mais pas conçus spécialement. Les travaux des auteurs suivants ont été faits avec de tels appareils : BURTON et al. $(1958,1977)$, Busta (1967), Cerf (1969), Cerf et Hermier (1973), Cerf (1977), Galesloot (1956), Horak (1980), Hostettler (1965), Hunter (1972), Konietzko et Reuter (1982), Lindgren et Swartling (1963), Martin et Harper (1964), Neaves et JARVis (1978), OQuendo et al. (1975), SHIH et al. (1980), Torres et Hedrick (1969, 1971), WANG et al. (1964).

Les appareils spéciaux sont conçus soit sans distribution des temps de séjour (DTS), soit avec une DTS connue. Les appareils courants ont tous une DTS. Burton (1958) a montré que la DTS devait être connue, ainsi que le profil de température, si l'on veut pouvoir caractériser convenablement la thermorésistance 
des spores en passant par un calcul approprié. Or la mesure de la DTS est difficile. Si elle est mal faite, il sera montré plus loin que l'interprétation des résultats peut être faussée.

Le travail expérimental dans un équipement à écoulement continu présente deux inconvénients notables :

- il demande de grandes quantités de spores;

- il nécessite de concevoir un dispositif de prélèvement aseptique, au moins si l'on travaille avec des spores de mésophiles.

\section{Dénombrement des survivants}

Il a été rappelé plus haut (paragraphe I-C) que les spores à prendre en considération sont celles qui sont capables de se développer dans le lait et de le déstabiliser. Ce n'est pas le cas de toutes les souches de laboratoire citées dans le tableau I. Ashton et al. (1968) ont démontré que la croissance de la souche B. stearothermophilus 1518 est inhibée du fait de la chélation du calcium et du magnésium par la caséine chauffée. Ceci se produit dans le lait cru et dans le lait UHT, mais pas dans le lait stérilisé en bouteille.

C'est pourquoi, si l'on veut connaitre le nombre total de survivants et pas seulement le nombre de survivants capables d'altérer le lait, il faut faire les dénombrements soit sur boîte de milieu nutritif gélosé, soit par la technique du nombre le plus probable (MPN) en milieu nutritif liquide ou dans du lait complémenté avec des cations (SOARes de Melo et al., 1973).

La sélection du meilleur milieu de dénombrement n'est pas simple. En effet, il a été démontré que le traitement UHT endommage le(s) système(s) de germination des spores. Le milieu de dénombrement doit donc être additionné de substances judicieusement choisies, telles que le dipicolinate de calcium pour B. subtilis, le lysozyme pour Clostridium perfringens, etc. (ADAMs, 1978 ; FoegEDING et Busta, 1981). En l'absence de données précises sur les souches étudiées, il est usuel d'ajouter de l'amidon au milieu de dénombrement des spores survivantes pour tenter d'obvier ce problème de lésion des systèmes de germination (par exemple Hermier et al., 1975).

\section{Interprétation des résultats}

\section{A. Flores naturelles}

Si l'on porte sur un graphique en ordonnée le logarithme du temps de destruction thermique et en abscisse la température, on obtient des droites pour ces flores naturelles comme avec des souches pures (HoraK, 1980; DuQueT et al., 1986). Ceci indique que les flores sont homogènes. Ces courbes sont donc très faciles à utiliser pour calculer les combinaisons température-temps assurant une efficacité stérilisatrice requise. 


\section{Courbes de survie}

\section{B. Souches de laboratoires}

Le seul cas où ces souches sont faciles à utiliser est celui où elles ont des courbes de survie rectilignes (relation linéaire entre le logarithme de la concentration en survivants et le temps). Les déviations de la linéarité ont déjà été mentionnées. Ce sont l'activation (qui correspond à un délai avant l'établissement de la constante de vitesse de destruction), la concavité vers le haut et la traînée (CERF, 1977).

Les souches suivantes ont des courbes de survie non linéaires : B. subtilis 786 (Burton, 1958), B. stearothermophilus TH 24 (Burton et al., 1977), C. botulinum, souches de type A et B (NeAves et JARVIS, 1978). De nombreux auteurs ne s'assurent pas de la linéarité.

\section{Effet de la température}

Jusqu'ici, l'effet de la température a été décrit au moyen du paramètre $\mathrm{z}$, inverse de la pente des droites obtenues en portant, en fonction de la température, le logarithme soit du temps de réduction décimale D soit du TDT, temps à partir duquel il reste moins de un survivant par tube en moyenne.

Ces droites sont appelées respectivement courbe fantôme de TDT et courbe de TDT.

Or beaucoup de phénomènes biologiques peuvent être décrits par l'équation thermodynamique d'Arrhenius, qui établit une relation linéaire entre le logarithme de $\mathrm{D}$ et l'inverse de la température absolue et permet le calcul de l'énergie d'activation $E_{a}$. On peut légitimement penser que cette relation pourrait s'appliquer à la destruction des spores. En raison de la dispersion des résultats expérimentaux, l'hypothèse ne peut être testée que si les essais sont faits sur un intervalle de température supérieur à $15 \mathrm{~K}$. Peu de travaux ont été faits sur un tel intervalle. Les uns dénombrent la validité des courbes de TDT :

$\begin{array}{ll}\text { - Pflug et Esselen (1953) } & 36 \mathrm{~K}\left(113 \text { à } 149^{\circ} \mathrm{C}\right) \\ \text { - Oquendo et al. (1975) } & 28 \mathrm{~K}\left(120 \text { à } 148^{\circ} \mathrm{C}\right) \\ \text { - KonietzKo et Reuter (1980) } & 27 \mathrm{~K}\left(120 \text { à } 147^{\circ} \mathrm{C}\right) \\ \text { - Srimani et al. }(1980) & 45 \mathrm{~K}\left(100 \text { à } 145^{\circ} \mathrm{C}\right)\end{array}$

Les autres démontrent la validité de la relation d'Arrhenius :

- WANG et al. (1964)

- Burton et al. (1977).

- Neaves et JaRvis (1978)

Miller et KANDLER (1967) trouvent une relation différente des deux précédentes : $\mathrm{z}$ augmente fortement avec la température. Mais ces auteurs ne tenaient pas compte de l'équilibrage des températures dans les tubes. Leurs résultats ne peuvent donc être retenus.

Les preuves en faveur de l'un ou l'autre des deux types d'interprétation conduisent à des convictions égales concernant l'un ou l'autre type. Il n'est pas possible de trancher. On recommandera seulement d'utiliser l'interprétation la mieux adaptée aux circonstances (CERF, 1985).

La présentation des résultats d'essais en écoulement continu peut être faite avec une courbe d'efficacité stérilisatrice (Hermier et al., 1975). Celle-ci est obtenue en portant en ordonnée le logarithme de l'efficacité stérilisatrice en fonction 
de la température. Rappelons que $\mathrm{E}=\log \mathrm{Co} / \mathrm{C}$ (Co concentration initiale des spores, C concentration des spores survivantes) (Galesloot, 1956). On obtient une droite d'équation :

$$
\log \mathrm{E}=\mathrm{T} / \mathrm{z}_{\mathrm{app}}+\mathrm{c}
$$

( $\mathrm{T}$ température, c une constante). Il est important de noter que c'est $\mathrm{z}_{\mathrm{app}}$, une valeur apparente de $\mathrm{z}$, et non la vraie valeur de $\mathrm{z}$, qui intervient dans cette équation (CERF, 1979). Si le besoin s'en fait sentir, on peut obtenir parfois une courbe d'efficacité stérilisatrice mieux ajustée avec l'équation (ici les abscisses sont $1 / \mathrm{T}$ ) :

$$
\log \mathrm{E}=\mathrm{E}_{\mathrm{a} . \mathrm{app}} / 19 \mathrm{~T}+\mathrm{d}
$$

(T température absolue, $\mathrm{d}$ une constante). Cette fois c'est une valeur apparente de l'énergie d'activation qui intervient dans l'équation. Elle est exprimée en joules par mole. Le chiffre 19 (J/mol.K) tient compte de la constante des gaz parfaits.

Les courbes d'efficacité stérilisatrice permettent de choisir au mieux la consigne de température à donner au stérilisateur, et de connaître, sans calcul supplémentaire, l'influence d'une variation de la température de stérilisation sur l'efficacité stérilisatrice.

\section{Effet de la vitesse de variation de température}

Brown et MeLling (1971) notaient : «Les effets de la soudaineté du changement de température et de son amplitude ont peu attiré l'attention, sauf pour le phénomène de refroidissement brusque. Il se pourrait bien que, comme cela peut se produire lors du refroidissement, la rapidité du chauffage endommage d'abord la membrane plutôt qu'un autre système. » Cette hypothèse et les résultats des premiers essais avec $C$. tyrobutyricum (accroissement de $\mathrm{z}$ de $10 \mathrm{~K}$ en tubes de TDT à $12 \mathrm{~K}$ en capillaires à $13 \mathrm{~K}$ par injection dans la vapeur) (CERF, 1969 ; Cerf et Hermier, 1973) ont incité à examiner de près si un choc thermique pourrait modifier la thermorésistance de spores. De fait, dans le cas de spores de $B$. coagulans 604 chauffées par injection directe dans la vapeur, il y a un effec de choc thermique qui dépend de l'amplitude du saut de température (DAuDIN et Cerf, 1977). Neaves et Jarvis (1978) ont observé ce même résultat avec des spores de $C$. botulinum de type A et B. Toutefois, ils l'attribuaient non à l'amplitude de variation de température, mais à sa vitesse. En revanche, STADHOUDERS et al. (1980) n'ont pas observé d'effet de choc thermique en chauffant des spores de $B$. cereus. Mais dans leur expérience, réalisée avec un échangeur tubulaire, la variation de température n'était pas soudaine.

A la connaissance de l'auteur, seulement deux autres articles concernent l'effet de choc thermique. Ils portent sur des cellules végétatives :

- Staphylococcus aureus a le même TDT qu'il soit chauffé en tube ou en capillaires (ZoTTOLA et JEZESKI, 1969);

- Escherichia coli injecté dans un excès de liquide chauffé est détruit plus vite que si on le chauffe progressivement (Tsuchido et al., 1974).

Lorsqu'il y a un effet de choc thermique dans un appareil UHT, il est pris en compte par un essai réalisé au moyen de cet appareil en écoulement continu. Dans les essais de Hermier et al. (1975) et Neaves et Jarvis (1978), il se traduit par :

- une augmentation de D aux températures les plus faibles;

- une augmentation apparente de $\mathrm{z}$. 
Mais, pour les essais de DAUdin et CERF (1977), il faut conclure apparemment que $\mathrm{D}$ décroîtra plus aux températures les plus élevées où le choc est plus violent, et par conséquent $\mathrm{z}$ diminuera. La contradiction provient d'une maîtrise incomplète des conditions expérimentales, dont l'explication est fournie par le paragraphe suivant.

\section{Effet de la distribution des temps de séjour (DTS)}

La raison pour laquelle les courbes d'efficacité stérilisatrice ne donnent accès qu'à des valeurs apparentes de $\mathrm{z}$ ou de $\mathrm{E}_{\mathrm{a}}$ est simplement la suivante : leur équation a été écrite en supposant que le temps de chauffage est constant sur tout l'intervalle de température. C'est une approximation. En effet, du fait de la DTS, tout se passe comme si le temps de séjour moyen diminue lorsque la température augmente, jusqu'à rejoindre la valeur du temps de séjour minimum (CERF, 1979). Toutefois, cette critique ne retire rien au caractère pratique des courbes d'efficacité stérilisatrice ; elle est néanmoins importante dans la mesure où elle explique complètement la contradiction signalée à la fin du paragraphe précédent.

La DTS est plus facile à mesurer lorsque le liquide passant dans un appareil est de l'eau plutôt que du lait. Mais, du fait de sa viscosité plus élevée, on obtient une DTS différente avec le lait (Heppell, 1985 a), et donc une mauvaise interprétation des résultats de thermorésistance dans ce produit.

\section{Conclusion}

Pour avoir des résultats entièrement cohérents, l'ensemble des paramètres expérimentaux doivent donc être contrôlés de façon rigoureuse : HePpelL (1985 b) donne le premier exemple de résultats obtenus à la suite d'une démarche qui tient compte de toutes les mises en garde énumérées dans le présent article. Ses résultats, qui ont demandé un travail considérable, ne comportent aucune contradiction.

Il est donc possible d'étudier avec la plus grande rigueur les effets des traitements à ultra-haute température sur les spores bactériennes de souches de laboratoire. On pourrait par exemple explorer avec profit le phénomène de choc thermique. Mais, ainsi qu'il a été exposé, ces souches ne sont pas représentatives de la flore naturelle des laits utilisés comme matières premières. Aussi, lorsqu'on désire optimiser un appareil UHT dans une laiterie donnée, il semble plus rapide et plus sûr d'utiliser l'une des techniques présentées au paragraphe I-A, et cela à plusieurs reprises au cours d'une année. Les températures et les durées de chambrage pourront ensuite tenir compte des variations saisonnières, et rendront possible un fonctionnement optimal, avec comme objectif : chauffer le lait comme il faut, mais pas plus. Ceux qui s'en donneront la peine constateront vite qu'on est loin de cette situation dans la majorité des usines. 


\section{Références bibliographiques}

ADAMS D.M., 1973. Inactivation of Clostridium perfringens type A spores at ultra-hightemperatures. Appl. Microbiol., 26, 282-287.

Adams D.M., 1978. Heat injury of bacterial spores. Adv. Appl. Microbiol., 23, 245-261.

ADAMS D.M., BuSTA F.F., 1972. Ultra-high-temperatures activation of a low-temperature Bacillus subtilis spore germination system. Appl. Microbiol.,24, 418-423.

ANonymous. 1967. Temperatur und Zeit-Abhängigkeit der Sporenabtötung im Bereich der Ultrahocherhitzung. Wiss. Jahresbericht, 10, Weihenstephan, 56.

Ashton D.H., Busta F.F., Warren J.A., 1968. Relief of casein inhibition of Bacillus stearothermophilus by iron, calcium and magnesium. Appl. Microbiol., 16, 628-635.

Ashron T.R., 1966, Control methods applied to aseptic milk production. Dairy Ind., 31, $480-482$.

BARACH J.T., FLoWERS R.S., ADAMS D.M., 1975, Repair of heat-injured Clostridium perfringens spores during outgrowth. Appl. Microbiol., 30, 873-875.

Bottazzi V., Dellaglio F., CoRradini C., 1966. Ricerche sul latte sterilizzato. 1. Effizienza di sterilizzazione di un impianto UHT Steriplak a scambio indiretto di calore. Sci. Tec. Latt. Casearia, 17, 133-142.

BRown M.R.W., MelLING J., 1971. Inhibition and destruction of microorganisms by heat. In : Inhibition and destruction of the microbial cell, 32. Hugo ed., Academic Press, Londres.

BURTON H., 1958. An analysis of the performance of an ultra-high-temperature milk sterilizing plant. I. Introduction and physical measurements. J. Dairy Res., 25, 75-84.

BURTON H., 1958. An analysis of the performance of an ultra-high-temperature milk sterilizing plant. II. Calculation of the bacterial effectiveness. I. Dairy Res., 25, 324-337.

BURTON H., 1970. Comparison of milks processed by the direct and indirect methods of ultra-high-temperature sterilization. III. A note on the results for spore destruction obtained with an experimental ultra-high-temperature milk sterilizer. J. Dairy Res., $37,227-231$.

Burton H., Franklin J.G., Williams D.J., Chapman H.R., Harrison A.J.W, Clegg L.F.L., 1958. An analysis of the performance of an ultra-high-temperature milk sterilizing plant. III. Comparison of experimental and calculated sporicidal effects for a strain of Bacillus subtilis. J. Dairy Res., 25, 338-343.

Burton H., Franklin J.G., Williams D.J., Chapman H.R., Harrison A.J.W. Clegg L.F.L., 1958. An analysis of the performance of an ultra-high-temperature milk sterilizing plant. IV. Comparison of experimental and calculated sporicidal effects for a strain of Bacillus stearothermophilus. J. Dairy Res., 26, 221-226.

Burton H., Perkin A.G., Davies F.L., UnDerwood H.M., 1977. Thermal death kinetics of Bacillus stearothermophilus spores at ultra-high-temperature. III. Relationship between data from capillary tube experiments and from UHT sterilizers. I. Food Technol, $12,149-161$.

Busta F.F., 1967. Thermal inactivation characteristics of bacterial spores at ultra-hightemperatures. Appl. Microbiol., 15, 640-645.

Busta F.F., ADAMS D.M., 1972. Identification of a germination system involved in the heat injury of Bacillus subtilis spores. Appl. Microbiol., 24, 412-417.

CERF O., 1969. Les Clostridium des produits laitiers : problèmes posés par leur numération. Mesure de leur thermorésistance. Thèse, Université de Caen.

CERF O., 1977. Tailing of survival curves of bacterial spores. J. Appl. Bacteriol., 42, 1-19.

CERF O., 1979. Relationship between temperature and sterilizing efficiency of continuous heat treatments of equal duration. In : Food Microbiology and Technology. Jarvis, Christian and Michener ed, Medicina Viva Servizio Congressi, Parme, 295-307. 
CERF O., 1985. Product quality and shelf life. In : Aseptic Processing and Packaging of Foods. Université de Lund et The Swedish Food Institute, 236-253.

Cerf O., HERMIER J., 1973. Thermorésistance anormale de spores bactériennes chauffées par injection directe dans la vapeur. Lait, 53, 23-39.

DanCKWERTS P.V., 1953. Continuous-flow system (distribution of residence time). Chem. Eng. Sci., 1, 1-13.

DAUdin J.D., Cerf O., 1977. Influence des chocs thermiques sur la destruction des spores bactériennes par la chaleur. Lebensmwiss. Technol., 10, 203-207.

Davies F.L., Underwood H.M., Perkin A.G., Burton H., 1977. Thermal death kinetics of Bacillus stearothermophilus spores at ultra-high-temperatures. I. Laboratory determination of temperature coefficients. J. Food Technol., 12, 115-129.

Duquet J.P., Trouvat A., Mouniqua A., Odet G., Cerf O., 1986. Les spores thermorésistantes du lait utilisé pour la fabrication de laits de longue conservation. Lait, accepté pour publication.

EdWARdS J.L., Jr., Busta F.F,, SPECK M.L., 1965. Thermal inactivation characteristics of Bacillus subtilis spores at ultra-high-temperatures. Appl. Microbiol., 13, 851-857.

Foegeding P.M., Busta F.F., 1981. Bacterial spore injury - an update. J. Food Prot., 44, 776-786.

Franklin J.G., Underwood H.M., Perkin A.G., Burton H., 1970. Comparison of milks processed by the direct and indirect methods of ultra-high-temperature sterilization, II. The sporicidal efficiency of an experimental plant for direct and indirect processing. J. Dairy Res., 37, 219-226.

Franklin J.G., Williams D.J., ChapMan H.R., Clegg L.F.L., 1958. Methods of assessing the sporicidal efficiency of an ultra-high-temperature milk sterilizing plant. II. Experiments with suspensions of spores in milk. J. Bacteriol,, 21, 47-50.

Franklin J.G., Williams D.J., ClegG L.F.L., 1958. Methods of assessing the sporicidal efficiency of an ultra-high-temperature milk sterilizing plant. III. Laboratory determination of the heat resistance of spores of Bacillus subtilis in water and in milk. J. Appl. Bacteriol, 21, 51-57.

GALESLOOT T.E., 1956. A simple model of estimating the bacteriological effect of sterilizing processes for milk, applied to the sterilization of milk in ultra-high-temperature short time (UHTST) and " in bottle " sterilizers. Neth. Milk Dairy J., 10, 79-100.

Heppell N.J., 1985 a. Comparison of the residence time distributions of water and milk in an experimental sterilizer. J. Food Eng., 4, 71-84.

HEPPELL N.J., 1985 b. A comparison between the measured and predicted sterilization performance of a laboratory-scale, direct heated UHT plant. J. Dairy Res., sous presse.

Hermier J., Begue P., Cerf O., 1975. Relationship between temperature and sterilizing efficiency of heat treatments of equal duration. Experimental testing with suspensions of spores in milk heated in an ultra-high-temperatures sterilizer. J. Dairy Res., 42, 437-444.

Hermier J., Verge J., Grosclaude G., 1959. Détermination de la durée de chauffage dans un appareil de stérilisation du lait à très haute température. Lait, 39, 20-32.

HoRAK F.P., 1980. Ueber die Reaktionskinetik der Sporenabtötung und chemicher Veränderungen bei der thermischen Haltbarmachung von Milch zur Optimierung von Erhitzungsverfahren. Thèse, Université de Technologie de Munich.

Hostettler H., 1961. Remplissage aseptique du lait upérisé en emballage Tetra Pak. Laitier Romand. J. Suisse Lait, 38 (46), 1-12.

HUNTER G.M., 1972. Continuous sterilization of liquid media containing suspended particles. Food Technol. Aust., 24 (4), 158-159, 162, 164-165.

KessLer H.G., 1981. Food Engineering and Dairy Technology, Verlag A. Kessler, Freising.

KonIETZKo M., REuTER H., 1980. Abtötung von Mikroorganismen während des UHTProzesses am Beispiel von Bacillus stearothermophilus. Milchwissenschaft, 35, 274-275.

LINDGREN B., SWARTLING P., 1963. The sterilizing efficiency of the Alfa-Laval Vacu-Therm Instant Sterilizer. Milk Dairy Res. Rep. Alnarp, $\mathrm{n}^{\circ} 69$. 
MARTIN J.H., HARPER W.J., 1964. Effect of ultra-high-temperatures on the destruction of bacterial spores. J. Dairy Sci., 47, 667-668.

Milleer I., KANDLER O., 1967. Temperature und Zeit-Abhängigkeit der Sporenabtötung im Bereich der Ultrahocherhitzung. Milchwissenschaft, 22, 689-691.

NeAves P., JARVIS B., 1978. Thermal inactivation kinetics of bacterial spores at ultra-hightemperatures, with particular reference to Clostridium botulinum. B.F.M.I.R.A. Res. Rep., $\mathrm{n}^{\circ} 280$ et 286.

Neaves P., JaRvis B., 1979. Thermal inactivation kinetics of bacterial spores at ultra-hightemperatures, with particular reference to Clostridium botulinum. Second report. B.F.M.I.R.A. Res. Rep., $\mathrm{n}^{\circ} 326$.

Odet G., Cerf O., Chevillotte J., Douard D., Glllis J.C., Helaine E., Lignac J., 1985. La maîtrise de la qualité du lait stérilisé UHT, A.P.R.I.A., Paris.

Oouendo R., Valdiviesco L., Stahl R., Loncin M., 1975. Versuchsanlage zu thermiche Inaktivierung von Mikroorganismen beiTemperaturen über $120^{\circ} \mathrm{C}$ und vernachlässigbarer Aufheiz und Abkuhlphase. Lebensm. wiss. Technol., 8, 181-182.

Osaka K., Shimamura S., Ando K., Kuniyasu C., 1968. Utilization of milk containing Bacillus anthracis using the ultra-high-temperatures process (en japonais). Bull. Nat. Inst. Anim., Health, Tokyo, 24-25.

PERKIN A.G., 1974. A laboratory-scale ultra-high-temperature milk sterilizer for batch operation. J. Dairy Res., 41, 55-63.

Perkin A.G., Burton H., Underwood H.M., Davies F.L., 1977. Thermal death kinetics of Bacillus stearothermophilus spores at ultra-high-temperatures. II. Effect of heating period on experimental results. J. Food Technol., 12, 131-148.

Pflug I.J., Esselen W.B., 1953. Development and application of an apparatus for study thermal resistance of bacterial spores and thiamine at temperature above $250^{\circ} \mathrm{F}$. Food Technol., 7, 237-241.

SHIH S.C., CUevas R., Porter V.L., Cheryan M., 1982. Inactivation of Bacillus stearothermophilus spores in soybean water extracts at ultra-high-temperatures in a scraped-surface heat exchanger. I. Food Prot., 45, 145-149.

Soares de Melo R., Cerf O., Hermier J., 1973. Sélection d'une souche de Bacillus thermophile pour la mesure de l'efficacité stérilisatrice des installations UHT. Lait, 53, 413-429.

SPECK M.L., BUSTA F.F., 1968. Sterilization and aseptic parakaging of milk products. Microbiological tests. J. Dairy Sci,, 51, 1146-1151.

SRIMANi B., Stahl R., LoNCIN M., 1980. Death rates of bacterial spores at high-temperratures. Lebensm. Wiss. Technol., 13, 186-189.

Stadhouders J., HuP G., Langeveld L.P.M., 1980. Some observations on the germination, heat resistance and outgrowth of fast-germinating and slow-germinating spores of Bacillus cereus in pasteurized milk. Neth. Milk Dairy J., 34, 215-228.

Thome K.E., Samuelsson E.G., Holm S., 1964. Milk Dairy Res. Rep. Alnarp, n ${ }^{\alpha} 1970$.

TORRES-AN.JEL M.J., HEDRICK T.I., 1971. Spore removal by centifugation and its effect on ultrahigh-temperature sterilization of milk. J. Dairy Sci., 52, 898.

TORRES M.J., HEDRICK T.I., 1971. Spore removal by centrifugation and its effect on ultrahigh-temperature commercial sterilization of milk. J. Dairy Sci., 54, 326-330.

Tsuchido T., Takano M., Shibasaki I., 1974. Effect of temperature-elevating process on the subsequent isothermal death of Escherichia coli K 12. J. Ferment. Technol., 52, 788-792.

WANG D.I.C., Scharer J., HuMPhReY A.E., 1964. Kinetics of death of bacterial spores at elevated temperatures. Appl. Microbiol., 12, 451-454.

Williams D.J., Franklin J.G., Chapman H.R., Clegg L.F.L., 1957. Methods of assessing the sporicidal efficiency of an ultra-high-temperatures milk sterilizing plant. I. Experiment with suspensions of spores in water. J. Appl. Bacteriol., 20, 43-49.

ZoTrola E.A., JEZESKI J.J., 1969. Comparisons of short-time holding procedures to determine thermal resistance of Staphylococcus aureus. J. Dairy Sci., 52, 1855-1857. 